\title{
$\mathbf{P}_{\text {ontifícia }} \mathbf{U}_{\text {niversidade }} \mathbf{C}_{\text {atólica }}$ \\ DO RIO DE JANEIRO PÚC
}

Rafael de Sequeira Baptista Ferraz

\begin{abstract}
Estimativa de Preços de Contratos Futuros Sobre Petróleo Utilizando o Método do Filtro de Kalman
\end{abstract}

Dissertação de Mestrado

Dissertação apresentada ao Programa de PósGraduação em Engenharia de Produção da PUC - Rio como requisito parcial para obtenção do título de Mestre em Engenharia de Produção.

Orientador: Prof. Tara Keshar Nanda Baidya Coorientador: Prof. Fernando Antonio Lucena Aiube

Rio de Janeiro, 30 de Junho de 2009 


\title{
$\mathbf{P}_{\text {ontifícia }} \mathbf{U}_{\text {niversidade }} \mathbf{C}_{\text {atólica }}$

Rafael de Sequeira Baptista Ferraz

\section{Estimativa de Preços de Contratos Futuros sobre Petróleo Utilizando o Método do Filtro de Kalman}

\begin{abstract}
Dissertação apresentada como requisito parcial para obtenção do título de Mestre pelo Programa de PósGraduação em Engenharia de Produção da PUC-Rio. Aprovada pela Comissão Examinadora abaixo assinada.
\end{abstract}

Prof. Tara Keshar Nanda Baidya Orientador Departamento de Engenharia Industrial - PUC-Rio

Prof. Fernando Antônio Lucena Aiube Coorientador Departamento de Engenharia Industrial - PUC-Rio

Prof. Paulo Henrique Soto Costa Departamento de Engenharia Industrial - PUC-Rio

Prof. Florival Rodrigues de Carvalho Departamento de Engenharia Química - UFPE

Sr. Krongnon Wailamer de Souza Regueira Consultor Autônomo

Prof. José Eugenio Leal Coordenador Setorial do Centro Técnico Científico - PUC-Rio 
Todos os direitos reservados. É proibida a reprodução total ou parcial do trabalho sem autorização da universidade, do autor e do orientador.

\section{Rafael de Sequeira Baptista Ferraz}

Graduou-se em Engenharia de Produção pela Pontifícia Universidade do Rio de Janeiro em 2006.

Ficha Catalográfica

Ferraz, Rafael de Sequeira Baptista

Estimativa de preços de contratos futuros sobre petróleo utilizando o método do filtro de Kalman / Rafael de Sequeira Baptista Ferraz ; orientador: Tara Keshar Nanda Baidya ; co-orientador: Fernando Antonio Lucena Aiube. 2009.

173 f. : il. (color.) ; $30 \mathrm{~cm}$

Dissertação (Mestrado em Engenharia Industrial)Pontifícia Universidade Católica do Rio de Janeiro, Rio de Janeiro, 2009.

Inclui bibliografia

1. Engenharia industrial - Teses. 2. Mercado futuro. 3. Processo estocástico. 4. Filtro de Kalman. 5. Séries temporais. 6. Previsão. I. Baidya, Tara Keshar Nanda. II. Aiube, Fernando Antonio Lucena. III. Pontifícia Universidade Católica do Rio de Janeiro. Departamento de Engenharia Industrial. IV. Título. 
À minha esposa Marília, à minha mãe Maria da Glória, ao meu pai Newton, aos meus irmãos André e Julia, à minha madrasta Rosana e à minha sogra Cláudia. 


\section{Agradecimentos}

Ao meu orientador Tara Baidya, professor do quadro principal do Departamento de Engenharia Industrial (DEI) da PUC-Rio, pela ajuda e cobrança exercidos no período de elaboração desta dissertação.

Ao meu coorientador Fernando Aiube, professor do DEI e funcionário da Petrobras, pelos ensinamentos e ajuda na implementação dos modelos.

Ao professor do DEI, Paulo Henrique Soto Costa, pelos auxílios e ensinamentos durante a graduação e o mestrado.

Ao professor Fabio Rodrigo Siqueira Batista, do DEI e do Centro de Pesquisas de Energia Elétrica (CEPEL), por aceitar participar da banca avaliadora desta dissertação.

Ao professor da Universidade Federal de Pernambuco e Superintendente da Agência Nacional de Petróleo e Gás Natural e Biocombustíveis (ANP), Florival Rodrigues Carvalho, por aceitar fazer parte da banca avaliadora.

Ao professor doutor Krongnon Wailamer de Souza Regueira e funcionário da Superintendência de Planejamento e Pesquisa da ANP pelo seu interesse em fazer parte da banca avaliadora desta dissertação.

À PUC-Rio e ao CNPq pela bolsa de mestrado concedida. 


\section{Resumo}

Ferraz, Rafael de Sequeira Baptista. Baidya, Tara Keshar Nanda. Estimativa de Preços de Contratos Futuros Sobre Petróleo Utilizando o Método do Filtro de Kalman. Rio de Janeiro, 2009. 173p. Dissertação de Mestrado - Departamento de Engenharia Industrial, Pontifícia Universidade Católica do Rio de Janeiro.

O Mercado Futuro adquire cada vez mais importância no cenário das Finanças Corporativas mundiais. $\mathrm{O}$ interesse principal das empresas neste segmento das finanças é a necessidade de proteção contra a volatilidade dos mercados financeiros. Neste sentido, uma das commodities mais negociadas é o petróleo. A dificuldade em precificar os contratos futuros do barril faz com que muitos modelos sejam criados para demonstrar a evolução dos preços ao longo do tempo. A utilização de processos estocásticos para representar possíveis trajetórias das séries temporais vem alcançando cada vez mais notoriedade, pois incorpora a aleatoriedade nas análises. O presente trabalho pretende testar a eficácia do modelo proposto na previsão do preço dos contratos futuros um passo à frente, ou seja, em estimar o preço para certa data na data imediatamente anterior. Neste sentido, o objetivo do estudo é coerente com o principal objetivo da análise de séries temporais que é construir modelos capazes de realizar previsões. Além disso, será estimado o preço à vista, variável a qual não é observável no mercado, e posteriormente serão confrontados os valores obtidos com uma proxy. O preço do mercado spot possui utilidade para os traders que necessitam obter o valor de um ativo que não é transacionado desta forma em bolsa. As estimativas dos parâmetros dos processos estocásticos serão feitas através de uma ferramenta estatística que passou a ser muito utilizada em modelos financeiros, o Filtro de Kalman. O procedimento consistirá em adotar um modelo de processo estocástico consagrado para uma série de preços de contratos futuros de uma commodity, estimando seus parâmetros e variáveis de estado com o Filtro, utilizando-o para previsão dos preços dos contratos futuros e para estimar o preço à vista, e posteriormente confrontando as estimativas e os valores reais coletados do mercado. Desta forma, se avaliará a capacidade do modelo em se adequar a novas mudanças estruturais na série. As ferramentas serão sempre explicitadas de 
maneira acessível, demonstrando cada passo tomado e sempre que possível fazendo paralelo com outros conhecimentos mais básicos.

\section{Palavras-chave}

Mercado Futuro; Processo Estocástico; Filtro de Kalman; Séries Temporais; Previsão 


\section{Abstract}

Ferraz, Rafael de Sequeira Baptista. Baidya, Tara Keshar Nanda. Estimation of Petroleum Future Contracts Using The Kalman Filter Method. Rio de Janeiro, 2009. 173p. MSc. Dissertation - Departamento de Engenharia Industrial, Pontifícia Universidade Católica do Rio de Janeiro.

The Future Market is becoming increasingly important in the global scenario of Corporate Finance. The main interest in this segment of finance is the need of being protected against the volatility of financial markets. Accordingly, one of the most traded commodity is oil. Because of difficulty in determine the value of future contracts on oil barrel, many models were created to demonstrate the evolution of their prices over time. The use of stochastic processes to represent possible trajectories of the time series is reaching more and more notoriety because it incorporates the randomness in the analysis. This study seeks to test the effectiveness of the proposed model in predicting the price of future contracts one step ahead, i.e. to estimate the price for a certain date on the preceding date. Consequently, the objective of the study is consistent with the primary objective of time series analysis that is to build models capable of forecasting. Furthermore, it will be estimated the spot price, variable that is not observable in the market, then the values obtained will be faced with a "proxy". The spot price is useful for traders who need to obtain the value of an asset that is not transacted in this way at the exchange. Estimates of the parameters of stochastic processes will be made through a statistical tool that has become widely used in financial models, the Kalman filter. The procedure is to adopt well known model of stochastic process for a series of prices of commodity futures contracts, estimating its parameters and state variables with the filter, using it to forecast the prices of future contracts and to estimate the spot price, and later comparing the estimates and the real values collected from the market. Thus, it will be evaluated ability of the model to fit the new structural changes in the time series. The tools will always be explained in an accessible way, demonstrating each step and where possible making parallel with other more basic knowledge. 


\section{Keywords}

Future Market; Stochastic Process; Kalman Filter; Time Series; Forecast 


\section{Sumário}

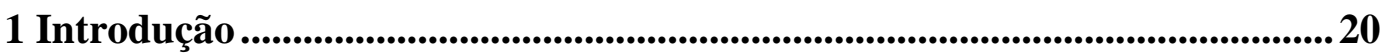

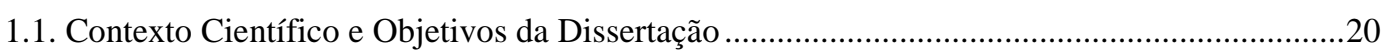

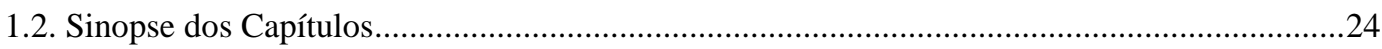

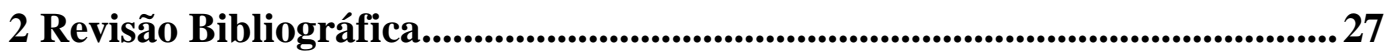

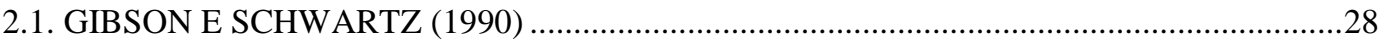

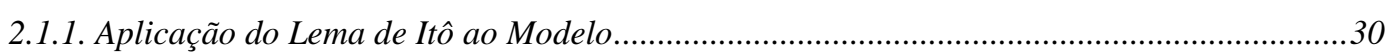

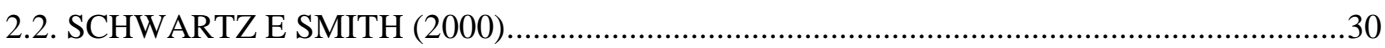

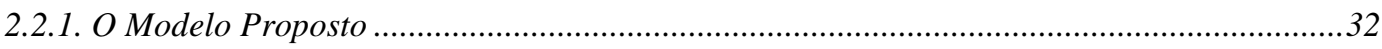

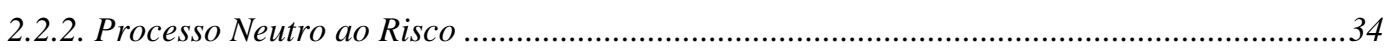

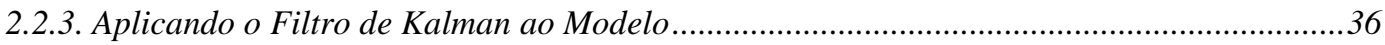

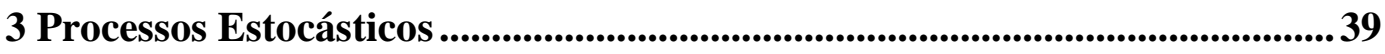

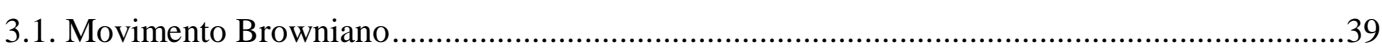

3.1.1. Movimento Aritmético Browniano.............................................................................. 40

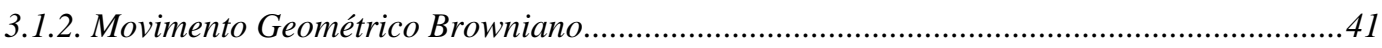

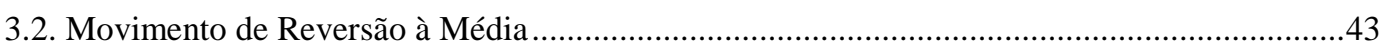

4 O Filtro de Kalman ...................................................................................................45

4.1. Comparação entre Mínimos Quadrados Ordinários e o Filtro de Kalman..............................46

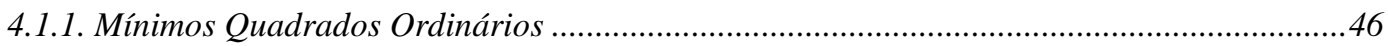

4.1.2. Algoritmo Recursivo de Mínimos Quadrados Ordinários....................................................47

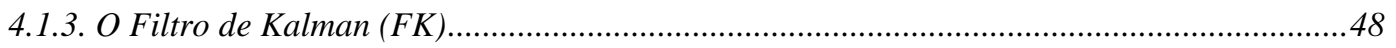

4.1.3.1. Equações de Atualização e Correção do Filtro de Kalman...................................................................51

4.1.3.2. Comparação Entre as Equações de Correção e Atualização

do FK e do Algoritmo Recursivo de MQO .................................................................................................52

4.1.3.3. O Algoritmo do Filtro de Kalman .................................................................................................5

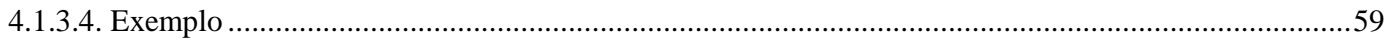

5 Filtro de Kalman Aplicado ao Modelo de Schwartz e Smith (2000).............65

5.1. Passagem dos Processos Estocásticos para o Filtro de Kalman .............................................65

5.1.1. Utilizando a Medida de Martingale Equivalente Para

Determinar a Relação Entre o Preço à Vista e Futuro .....................................................................66

5.1.2. Discretização do Modelo Para Utilizar O Filtro de Kalman ...............................................67

5.2. Aplicando a Estimativa de Máxima Verossimilhança ao Filtro de Kalman ...........................68

5.2.1. Maximização da Função de Verossimilhança de Observações com f.d.p. Normal...............69

5.2.2. Maximização da Função de Verossimilhança no Caso do Filtro de Kalman .......................70 
6 Implementação do Modelo e do Filtro de Kalman ............................................ 73

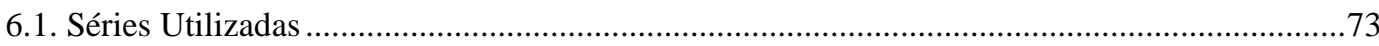

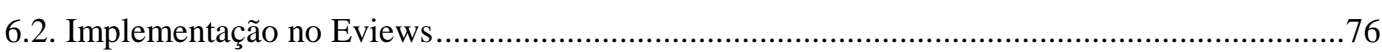

7 Apresentação dos Resultados ......................................................................79

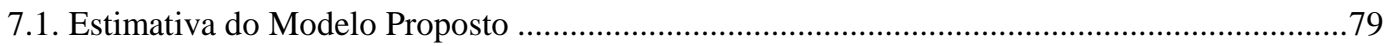

7.2. Ajuste das Séries de Contratos Futuros Estimadas e Reais ....................................................83

7.2.1. Gráficos de Contratos Estimados vs Observados....................................................................84

7.2.2. Gráficos dos Erros de Estimativa Um Passo a Frente .......................................................86

7.2.3. Análise dos Resíduos Gerados ............................................................................................. 88

7.3. Estimativas Para as Variáveis de Estado .............................................................................92

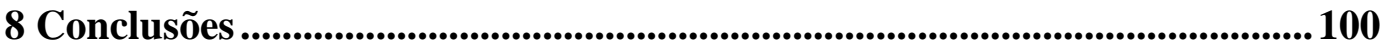

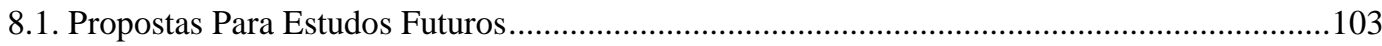

9 Referências ..............................................................................................105

10 Apêndice A - Aplicação do Lema de Itô ao Modelo de Gibson

Schwartz (1990) ...........................................................................................109

11 Apêndice B - Função Geradora de Momentos ...............................................113

11.1. Aplicação da Função Geradora de Momentos ao Modelo de S\&S (2000) .........................115

11.2. Aplicação da Função Geradora de Momentos ao Modelo de S\&S (2000)

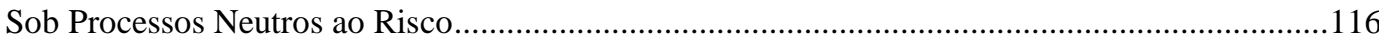

12 Apêndice C - Demonstrações das Propriedades Estatísticas dos

Processos Estocásticos Abordados .................................................................... 117

12.1. Demonstração da Evolução da Variância do Processo de Wiener no Tempo .....................117

12.2. Demonstração do Valor Esperado e da Variância do MGB ................................................119

12.3. Média, Variância e Outras Características do MRM .......................................................122

13 Apêndice D - Minimização do Erro Quadrático .............................................. 127

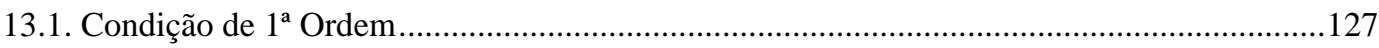

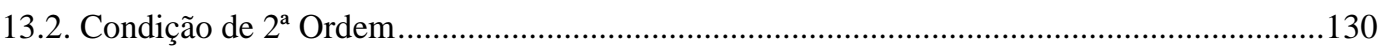

14 Apêndice E - Demonstração de que $b$ é o MELNT de $\beta$............................. 132

15 Apêndice F - Demonstração do Algoritmo Recursivo de MQO ............... 140

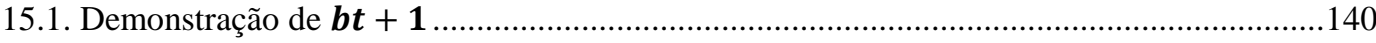

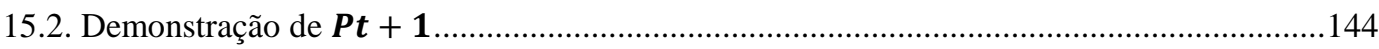

15.3. Relação Entre a Variância de bt e $\boldsymbol{P t}$............................................................................ 145 
16 Apêndice G - Demonstrações do Filtro de Kalman .................................... 147

16.1. Transformação das Equações do FK Para Equações com Constantes .................................147

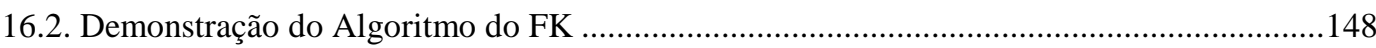

16.3. Atualização do Vetor de Estado Após a Observação ..........................................................151

16.3.1. Fatoração Triangular de Uma Matriz Simétrica Positiva Definida..................................155

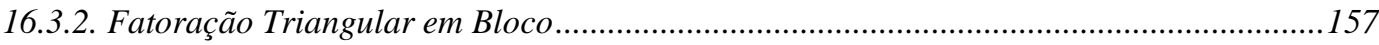

16.4. Maximização da Função de Verossimilhança Quando as

Observações se Distribuem Normalmente.

\section{Apêndice H - Demonstração do Lema de Inversão de Matrizes}

(Álgebra).

18 Apêndice I - Gráficos Para Análise do Ajuste do Modelo aos Dados

Reais 166

18.1. Gráficos de Ajuste dos Contratos Futuros Observados vs Filtrados..................................166

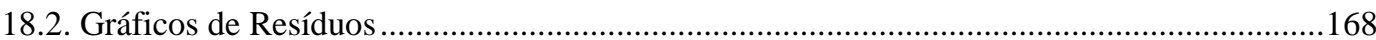

18.3. Análise qualitativa da Normalidade dos Resíduos .............................................................170 


\section{Lista de Figuras}

Figura 1 - Simulação Para Movimento Aritmético Browniano............................... 41

Figura 2 - Simulação Para Movimento Geométrico Browniano ............................ 42

Figura 3 - Simulação Para Movimento de Reversão à Média ................................ 44

Figura 4 - Ilustração Para o Algoritmo do FK na Data Um ..................................56

Figura 5 - Ilustração Para o Algoritmo do FK na Data Dois .................................5

Figura 6 - Algoritmo Para o Filtro de Kalman ...................................................58

Figura 7 - Linha do Tempo Para o Filtro de Kalman .............................................59

Figura 8 - Preço Observado vs Filtrado Para o Contrato F1 ................................ 84

Figura 9 - Preço Observado vs Filtrado Para o Contrato F5 ................................ 85

Figura 10 - Erros de Estimativa Para o Contrato F1 .............................................. 86

Figura 11 - Erros de Estimativa Para o Contrato F5 .............................................. 87

Figura 12 - Erros Percentuais Para o Contrato F1 ................................................. 88

Figura 13 - Distribuição dos Resíduos do Contrato F1 .........................................89

Figura 14 - Distribuição dos Resíduos do Contrato F5 .........................................90

Figura 15 - Estimativa Para o Desvio de Curto-Prazo............................................. 94

Figura 16 - Estimativa Para o Equilíbrio de Longo-Prazo .................................... 95

Figura 17 - Estimativa Para o Logaritmo do Preço à Vista .................................... 96

Figura 18 - Estimativa Para o Preço à Vista ......................................................... 96

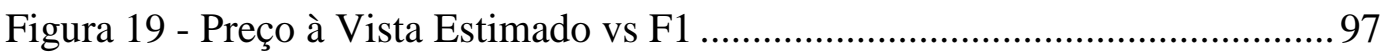

Figura 20 - Preço Observado vs Filtrado Para o Contrato F9 ............................. 166

Figura 21 - Preço Observado vs Filtrado Para o Contrato F13 ........................... 167

Figura 22 - Preço Observado vs Filtrado Para o Contrato F17 ........................... 168

Figura 23 - Erros de Estimativa Para o Contrato F9............................................ 168

Figura 24 - Erros de Estimativa Para o Contrato F13......................................... 169

Figura 25 - Erros de Estimativa Para o Contrato F17........................................ 170

Figura 26 - Distribuição dos Resíduos do Contrato F9 ....................................... 171

Figura 27 - Distribuição dos Resíduos do Contrato F13 ………......................... 172

Figura 28 - Distribuição dos Resíduos do Contrato F17 .................................... 173 


\section{Lista de Tabelas}

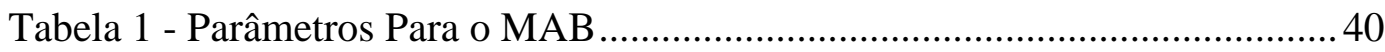

Tabela 2 - Parâmetros Para o MGB ....................................................................... 42

Tabela 3 - Parâmetros Para o MRM ...................................................................... 44

Tabela 4 - Nomenclatura Adotada Para os Contratos

Futuros em Função do Prazo Para a Expiração ...................................................... 74

Tabela 5 - Principais Estaatísticas das Séries de Contratos Futuros Selecionadas 75

Tabela 6 - Estimativa Para o Modelo Proposto ........................................................ 80

Tabela 7 - Comparação Entre as Estimativas Encontradas

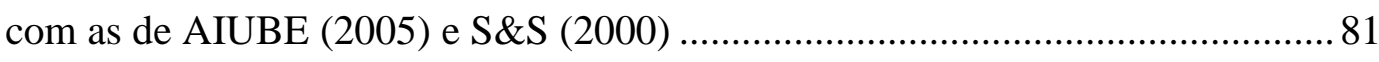

Tabela 8 - Estatísticas de Teste de Normalidade

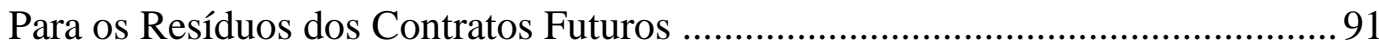

Tabela 9 - Estatísticas de Ajuste do Modelo aos Contatos Futuros ....................... 92

Tabela 10 - Estatísticas de Ajuste Entre o Preço à

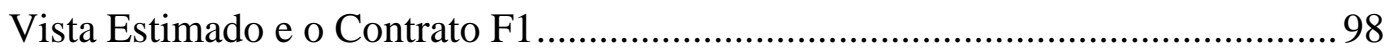

Tabela 11 - Estatísticas Médias de Ajuste do Modelo aos Contratos Futuros ...... 98 


\section{Lista de Símbolos e Abreviações}

a.a.

bbl(s)

E\&P

f.d.p.

FK

MME

MAB

MAE

MAPE

MB

MGB

MRM

MQO

NYMEX

RMSE

S\&S

US\$

WTI

*

$\boldsymbol{x}_{\boldsymbol{t}^{+}}$

$x_{t^{-}}$

$\boldsymbol{P}_{\boldsymbol{t}^{+}}$

$\boldsymbol{P}_{\boldsymbol{t}^{-}}$

$K_{t}$

$z_{t}$

$x_{t}$

$\boldsymbol{H}_{\boldsymbol{t}}$

$\boldsymbol{u}_{t}$

$\phi$

G
Ao ano

Volume em Barril ou Barris

Exploração e Produção de Petróleo

Função Densidade de Probabilidade

Filtro de Kalman

Medida de Martingal Equivalente

Movimento Aritmético Browniano

Erro Absoluto Médio

Erro Absoluto Médio Percentual

Movimento Browniano

Movimento Geométrico Browniano

Movimento de Reversão à Média

Mínimos Quadrados Ordinários

New York Mercantile Exchange

Raiz do Erro Quadrático Médio

Schwartz e Smith

Dólares Norte Americanos

West Texas Intermediate

Variável sob Precesso Neutro ao Risco

Vetor Calculado Após Observação da data $t$

Vetor previsto para a data $t$

Matriz de Covariâncias dos erros de previsão para o vetor de estado após obter-se a informação de $t$

Matriz Calculada Antes da Observação da data $t$

Vetor de Ganhos de Kalman calculado após observação de $t$

Vetor de variáveis observadas em $t$

Vetor de variáveis de estado estimado para $t$

Matriz de hiperparâmetros

Distúrbios aleatórios em $t$

Matriz que apresenta a evolução das variáveis de estado no tempo

Matriz da dinâmica dos erros das equações de estado 
$N\left(\mu, \sigma^{2}\right) \quad$ Distribuição Normal com Média $\mu$ e variância $\sigma^{2}$

$X_{t 2} \quad$ Valor Observado da Variável Independente Dois na Data $t$

$f(y, \theta) \quad$ f.d.p. onde $y$ representa os valores da série e $\theta$ os parâmetros

$L(\hat{\theta}, y) \quad$ Função de Verossimilhança 


\section{Lista de Equações}

$$
\begin{aligned}
& F=S e^{((r+u-\delta)(T-t))} \\
& B(S, \delta, \tau) \\
& \frac{d S}{S}=\mu d t+\sigma_{1} d z_{1} \\
& d \delta=k(\alpha-\delta) d t+\sigma_{2} d z_{2} \\
& \frac{1}{2} B_{S S} S^{2} \sigma_{1}{ }^{2}+\frac{1}{2} B_{\delta \delta} \sigma_{2}^{2}+B_{S \delta} S \rho \sigma_{1} \sigma_{2}+B_{S} S(r-\delta)+ \\
& B_{\delta}\left[k(\alpha-\delta)-\lambda \sigma_{2}\right]-B_{\tau}-r B=0 \\
& \ln \left(S_{t}\right)=\chi_{t}+\xi_{t} \\
& d \chi_{t}=-k \chi_{t} d t+\sigma_{\chi} d z_{\chi} \\
& d \xi_{t}=\mu_{\xi} d t+\sigma_{\xi} d z_{\xi} \\
& d z_{\chi} d z_{\xi}=\rho_{\chi \xi} d t \\
& E\left[\left(\chi_{t}, \xi_{t}\right)\right]=\left[e^{-k t} \chi_{0}, \xi_{0}+\mu_{\xi} t\right] \\
& \operatorname{Cov}\left[\left(\chi_{t}, \xi_{t}\right)\right]=\left[\begin{array}{cc}
\left(1-e^{-2 k t}\right) \frac{\sigma_{\chi}^{2}}{2 k} & \left(1-e^{-k t}\right) \frac{\rho_{\chi \xi} \sigma_{\chi} \sigma_{\xi}}{k} \\
\left(1-e^{-k t}\right) \frac{\rho_{\chi} \sigma_{\chi} \sigma_{\xi}}{k} & \sigma_{\xi}^{2} t
\end{array}\right] \\
& E\left[\ln \left(S_{t}\right)\right]=e^{-k t} \chi_{0}+\xi_{0}+\mu_{\xi} t \\
& \operatorname{Var}\left[\ln \left(S_{t}\right)\right]=\left(1-e^{-2 k t}\right) \frac{\sigma_{\chi}^{2}}{2 k}+\sigma_{\xi}^{2} t+2\left(1-e^{-k t}\right) \frac{\rho_{\chi \xi} \sigma_{\chi} \sigma_{\xi}}{k} \\
& \ln \left(E\left[S_{t}\right]\right)=e^{-k t} \chi_{0}+\xi_{0}+\mu_{\xi} t+\frac{1}{2}\left(1-e^{-2 k t}\right) \frac{\sigma_{\chi}^{2}}{2 k}+\sigma_{\xi}^{2} t+ \\
& 2\left(1-e^{-k t}\right) \frac{\rho_{\chi \xi} \sigma_{\chi} \sigma_{\xi}}{k} \\
& d \chi_{t}=\left(-k \chi_{t}-\lambda_{\chi}\right) d t+\sigma_{\chi} d z_{\chi}^{*} \\
& d \xi_{t}=\left(\mu_{\xi}-\lambda_{\xi}\right) d t+\sigma_{\xi} d z_{\xi}^{*} \\
& d z_{\chi}^{*} d z_{\xi}^{*}=\rho_{\chi \xi} d t \\
& E^{*}\left[\left(\chi_{t}, \xi_{t}\right)\right]=\left[e^{-k t} \chi_{0}-\left(1-e^{-k t}\right) \frac{\lambda_{\chi}}{k}, \xi_{0}+\mu_{\xi}^{*} t\right] \\
& \operatorname{Cov}^{*}\left[\left(\chi_{t}, \xi_{t}\right)\right]=\operatorname{Cov}\left[\left(\chi_{t}, \xi_{t}\right)\right] \\
& E^{*}\left[\ln \left(S_{t}\right)\right]=e^{-k t} \chi_{0}-\left(1-e^{-k t}\right) \frac{\lambda_{\chi}}{k}+\xi_{0}+\mu_{\xi}^{*} t \\
& \operatorname{Var}^{*}\left[\ln \left(S_{t}\right)\right]=\operatorname{Var}\left[\ln \left(S_{t}\right)\right] \\
& \ln \left(F_{T, 0}\right)=\ln \left(E^{*}\left[S_{T}\right]\right)
\end{aligned}
$$




$$
\begin{aligned}
& \ln \left(F_{T, 0}\right)=e^{-k T} \chi_{0}+\xi_{0}+A(T) \\
& A(T)=\mu_{\xi}^{*} T-\left(1-e^{-k T}\right) \frac{\lambda_{\chi}}{k}+\frac{1}{2}\left(\left(1-e^{-2 k T}\right) \frac{\sigma_{\chi}^{2}}{2 k}+\right. \\
& \left.\sigma_{\xi}^{2} T+2\left(1-e^{-k T}\right) \frac{\rho_{\chi \xi} \sigma_{\chi} \sigma_{\xi}}{k}\right) \\
& x_{t}=\phi x_{t-1}+G v_{t} \\
& {\left[\begin{array}{l}
\chi_{t} \\
\xi_{t}
\end{array}\right]=\left[\begin{array}{c}
0 \\
\mu_{\xi}^{*} \Delta t
\end{array}\right]+\left[\begin{array}{cc}
e^{-k \Delta t} & 0 \\
0 & 1
\end{array}\right]\left[\begin{array}{l}
\chi_{t-1} \\
\xi_{t-1}
\end{array}\right]+\left[\begin{array}{l}
v_{1 t} \\
v_{2 t}
\end{array}\right]} \\
& \operatorname{Cov}\left[\left(\chi_{\Delta t}, \xi_{\Delta t}\right)\right]=\left[\begin{array}{cc}
\left(1-e^{-2 k t}\right) \frac{\sigma_{\chi}^{2}}{2 k} & \left(1-e^{-k t}\right) \frac{\rho_{\chi \xi} \sigma_{\chi} \sigma_{\xi}}{k} \\
\left(1-e^{-k t}\right) \frac{\rho_{\chi \xi} \sigma_{\chi} \sigma_{\xi}}{k} & \sigma_{\xi}^{2} t
\end{array}\right] \\
& z_{t}=H_{t} x_{t}+u_{t} \\
& {\left[\begin{array}{c}
\ln F_{T_{1}} \\
\vdots \\
\ln F_{T_{n}}
\end{array}\right]=\left[\begin{array}{c}
A\left(T_{1}\right) \\
\vdots \\
A\left(T_{n}\right)
\end{array}\right]+\left[\begin{array}{cc}
e^{-k T_{1}} & 1 \\
\vdots & \vdots \\
e^{-k T_{n}} & 1
\end{array}\right]\left[\begin{array}{l}
\chi_{t} \\
\xi_{t}
\end{array}\right]+\left[\begin{array}{c}
u_{1 t} \\
\vdots \\
u_{n t}
\end{array}\right]} \\
& K_{t}=P_{t^{-}} H_{t}^{\prime}\left[H_{t} P_{t^{-}} H_{t}^{\prime}+R_{t}\right]^{-1}
\end{aligned}
$$

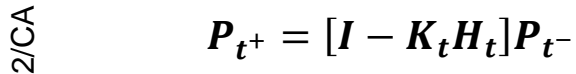

$$
\begin{aligned}
& x_{t^{+}}=x_{t^{-}}+K_{t}\left[z_{t}-H_{t} x_{t^{-}}\right] \\
& P_{(t+1)^{-}}=\phi P_{t^{+}} \phi^{\prime}+G Q G^{\prime} \\
& x_{(t+1)^{-}}=\phi x_{t^{+}} \\
& d x=\alpha d t+\sigma d z \therefore d z=\varepsilon \sqrt{d t} \therefore \varepsilon \sim N(0,1) \\
& E[d x]=E[\alpha d t+\sigma d z]=\alpha d t+\sigma E[d z]=\alpha d t \\
& \operatorname{Var}[d x]=\sigma^{2} d t \\
& d x \sim N\left(\alpha d t, \sigma^{2} d t\right) \\
& d x=a(x, t) d t+b(x, t) d z \\
& d x=\alpha x d t+\sigma x d z \\
& E\left[x_{t}\right]=x_{0} \mathrm{e}^{\alpha T} \\
& \operatorname{Var}\left[x_{t}\right]=x_{0}{ }^{2} \mathrm{e}^{2 \alpha T+\sigma^{2} T}-x_{0}{ }^{2} \mathrm{e}^{2 \alpha T}=x_{0}{ }^{2} \mathrm{e}^{2 \alpha T}\left(\mathrm{e}^{\sigma^{2} T}-1\right) \\
& d x=\eta(\bar{x}-x) d t+\sigma d z \\
& \operatorname{Var}\left[x_{t}\right]=\frac{\sigma^{2}}{2 \eta}\left(1-e^{-2 \eta t}\right) \\
& \boldsymbol{b}_{t}=\left(\boldsymbol{X}_{t}{ }^{\prime} \boldsymbol{X}_{\boldsymbol{t}}\right)^{-1} \boldsymbol{X}_{\boldsymbol{t}}{ }^{\prime} \boldsymbol{Y}_{\boldsymbol{t}} \\
& Y_{t}=\beta_{1}+\beta_{2} X_{t 2}+\beta_{3} X_{t 3}+\cdots+\beta_{k} X_{t k}+\varepsilon_{t} \\
& Y_{t}=X_{t} b_{t}+e_{t} \\
& b_{t+1}=b_{t}+K_{t+1}\left(Y_{t+1}-x_{t+1}^{\prime} b_{t}\right)
\end{aligned}
$$




$$
\begin{aligned}
& K_{t+1}=\frac{\left(X_{t}^{\prime} X_{t}\right)^{-1} x_{t+1}}{I+x_{t+1}^{\prime}\left(X_{t}^{\prime} X_{t}\right)^{-1} x_{t+1}} \\
& \boldsymbol{P}_{t}=\left(X_{t}^{\prime} X_{t}\right)^{-1} \\
& \boldsymbol{P}_{t+1}=P_{t}-P_{t} \frac{x_{t+1} x_{t+1}^{\prime}}{I+x_{t+1}^{\prime} P_{t} x_{t+1}} P_{t} \\
& f(y, \theta)=\prod_{t=1}^{T} f\left(y_{t}, \theta\right) \\
& \hat{\mu}=\frac{\sum_{t=1}^{T} y_{t}}{T} \\
& \widehat{\sigma^{2}}=\frac{\sum_{t=1}^{T}\left(y_{t}-\mu\right)^{2}}{T} \\
& E\left(\mathbf{z}_{t} \mid t-1\right)=\boldsymbol{H}_{t^{\prime}} \boldsymbol{x}_{\boldsymbol{t}^{-}} \\
& \boldsymbol{P}_{t^{-}}=E\left[\left(\boldsymbol{x}_{\boldsymbol{t}}-\boldsymbol{x}_{\boldsymbol{t}^{-}}\right)\left(\boldsymbol{x}_{\boldsymbol{t}}-\boldsymbol{x}_{\boldsymbol{t}^{-}}\right)^{\prime}\right] \\
& \boldsymbol{R}_{\boldsymbol{t}}=E\left[\boldsymbol{u}_{t} \boldsymbol{u}_{t}{ }^{\prime}\right] \\
& \boldsymbol{F}_{t}=\boldsymbol{H}_{\boldsymbol{t}} \boldsymbol{P}_{\boldsymbol{t}^{-}} \boldsymbol{H}_{\boldsymbol{t}}^{\prime}+\boldsymbol{R}_{\boldsymbol{t}} \\
& L(\boldsymbol{y} ; \boldsymbol{\theta})=-\frac{T}{2} \ln [2 \pi]-\frac{1}{2} \sum_{t=1}^{T}\left|\boldsymbol{F}_{t}\right|-\frac{1}{2} \sum_{t=1}^{T} \boldsymbol{v}_{t}^{\prime} \boldsymbol{F}_{t}^{-1} \boldsymbol{v}_{t}
\end{aligned}
$$

ญิ $\Delta t=\frac{1}{52}$

$$
R M S E=\sqrt{\frac{\sum_{i=1}^{n}\left(F_{o i}-F_{e i}\right)^{2}}{n}}
$$

$M A E=\frac{\sum_{i=1}^{n}\left|F_{o i}-F_{e i}\right|}{n}$

$$
M A P E=\frac{1}{n} \sum_{i=1}^{n}\left|\frac{F_{O i}-F_{e i}}{F_{o i}}\right|
$$

$J B=\frac{n}{6}\left(S^{2}+\frac{(K-3)^{2}}{4}\right)$ 